\title{
DIVISION IX / COMMISSION 30 / WORKING GROUP CATALOG OF ORBITAL ELEMENTS OF SPECTROSCOPIC BINARIES
}

\author{
CHAIR \\ VICE-CHAIR \\ MEMBERS
}

\author{
Dimitri Pourbaix \\ Andrew T. Young \\ Alan H. Batten, Francis C. Fekel, \\ William I. Hartkopf, Hugo Levato, \\ Nidia I. Morrell, Andrei A. Tokovinin, \\ Guillermo Torres, Stepane Udry
}

\section{TRIENNIAL REPORT 2006 - 2009}

The SB9 Working Group of Commission 30 aims at compiling the 9th Catalogue of Orbits of Spectroscopic Binaries. By definition, this is a never ending task as orbits of newly discovered systems keep appearing in the literature. Despite this, the working group tries to catch up with the delay as nothing was done in between 1989 when the 8th catalogue by Batten et al. and 2000 when the WG was settled. In 2006, at its business meeting, the WG decided to focus on the completeness of systems rather than on completeness of orbits. If the latter is a valuable objective, only the former is useful to any statistical investigation of spectroscopic binaries.

It was also decided that spectroscopic orbits of extrasolar planets should make it to the catalogue. Owing to the lack of manpower, to the instability of some planetary status, that task has been postponed so far. Another pending issue is the automatic grading scheme. In 2007, a student from the Université Libre de Bruxelles sought any computable quality indicator which could reproduce the original subjective grade listed in SB8 (and predecessors). No positive result has been achieved so far.

The main progress of SB9 over the past two years has focused on uploading papers with new systems, trying to be as complete as possible with some authors' series of papers (e.g. Carquillat, Griffin, Fekel, ...). 155 new systems have thus been uploaded (for 202 orbits in 44 papers). SB9 now contains 2839 systems, i.e., 1370 more than in the 8th catalogue. By the IAU XXVII General Assembly in 2009, we will try to upload the systems missing in order to double the sample of SB8.

Despite its spread in the community (assessed through ADS citations: 58 quotations to the release paper, Pourbaix et al. (2004, A\&A, 424, 727) and its efficiency to identify typographic bugs in some already published orbits, SB9 does not attracts orbits yet! Besides a very limited number of authors who spontaneously send their new orbits with all the relevant data, nobody sends his/her new solution nor let us know about the coming paper. That is a very unfortunate and frustrating situation because a little help from every author would make the whole community benefit from a better and more complete catalogue.

\author{
Dimitri Pourbaix \\ chair of the Working Group
}

